\title{
The whole-genome sequence analysis of Morchella sextelata
}

\author{
Mei-Han ${ }^{1}$, Qingshan-Wang ${ }^{1}$, Baiyintala ${ }^{2}$ \& Wuhanqimuge ${ }^{3 *}$
}

Morchella are macrofungi and are also called morels, as they exhibit a morel-like upper cap structure. Morels contain abundant essential amino acids, vitamins and biologically active compounds, which provide substantial health benefits. Approximately 80 species of Morchella have been reported, and even more species have been isolated. However, the lack of wild Morchella resources and the difficulties associated with culturing Morchella have caused a shortage in the morels available for daily consumption. Additionally, in-depth genomic and morphological studies are still needed. In this study, to provide genomic data for further investigations of culturing techniques and the biological functions of Morchella sextelata (M. sextelata), de novo genome sequencing was carried out on the Illumina HiSeq. 4000 platform using both the Illumina 150 and PacBio systems. The final estimated genome size of $M$. sextelata was $52.93 \mathrm{Mb}$, containing 59 contigs and a GC content of $47.37 \%$. A total of 9,550 protein-coding genes were annotated. In addition, the repeat sequences, gene components and gene functions were analyzed using various databases. Furthermore, the secondary metabolite gene clusters and the predicted structures of their products were analyzed. Finally, a genomic comparison of different species of Morchella was performed.

Morchella is a member of the Morchellaceae family in the Pezizales order of the Pezizomycetes class under the Ascomycota division in the fungi kingdom. Morchella has a beautiful cap with a honeycomb-like structure and a brown, yellow, black or pale color that looks similar to lamb tripe, giving it the name "morel". Morchella has been reported to have a high edible value because it contains sufficient essential amino acids, vitamins, mineral elements and proteins ${ }^{1,2}$.

Morchella was identified as early as 1882 and has rapidly expanded in recent years, not only because of its rich nutritional value but also because of its diverse biological functions, such as anti-inflammatory, anti-oxidative, antiviral, and antitumor effects ${ }^{3-5}$. Moreover, Morchella is also a great source of biomacromolecules, including polynucleotides, polysaccharides, proteins and small molecules ${ }^{4}$. Some metabolites prepared from Morchella have been used in tumor treatment ${ }^{4}$. Furthermore, some extracts from Morchella have been useful and helpful in the therapies of cancers, diabetes, and cardiovascular diseases ${ }^{6}$. Therefore, in recent years, Morchella has become more popular. However, naturally growing Morchella is very rare and expensive and thus insufficient to meet the demands of consumers ${ }^{7}$.

In the latest index of fungorum at http://www.indexfungorum.org/names/names.asp, 334 isolates of Morchella, including subspecies, have been reported. In the literature, approximately 80 species of Morchella have been identified since the beginning of this century ${ }^{7}$, and even more species have been discovered. Several species, $M$. rufobrunnea, M. sextelata, M. eximia and M. importuna, have been successfully cultivated in China ${ }^{8}$. The research on Morchella has increased dramatically in recent years and started to focus on the techniques of artificial planting and the biological functions of Morchella. However, there is still a lack of information about their genome to improve culturing techniques and to screen good species.

The genome sequence of $M$. conica $(48.2133 \mathrm{Mb})$, M. importuna (median total length of 2 assemblies: 49.967 Mb), M. septimelata (median total length of 3 assemblies: $49.9613 \mathrm{Mb}$ ), and M. eximia (total length $73.461 \mathrm{Mb}$ ) have recently been reported in NCBI GenBank. However, genetic information about M. sextelata has not yet been reported.

The M. sextelata isolate studied here was successfully cultivated in Inner Mongolia, China, using relatively simple techniques and low-cost materials. Interestingly, the M. sextelata isolate cultivated here was larger, and its output was higher than that of other morels cultivated in other areas (Fig. S1). To investigate the genetic information and provide the data for further study of the biological functions of $M$. sextelata, a de novo sequence analysis

${ }^{1}$ Inner Mongolia ZhongXing Agriculture and Animal Husbandry Development Co., Ltd., Ulanqab, China. ${ }^{2}$ Inner Mongolia Mang Lai Food Co., Ltd., Hohhot, China. Innovative Mongolian Pharmaceutical Preparations Laboratory of Inner Mongolia, Inner Mongolia International Mongolian Hospital, Hohhot, China. *email: gege_403@163.com 


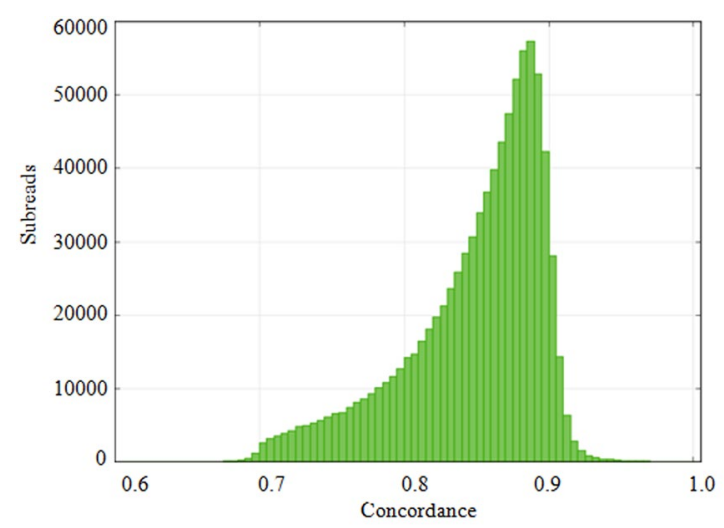

Concordance distribution of reads for $M$. sextelata genomic sequencing.

Figure 1. Horizontal coordinates represent the sequencing quality. The bars correspond to the left vertical coordinate, which shows the reads relevant to the quality of each sequencing data set.

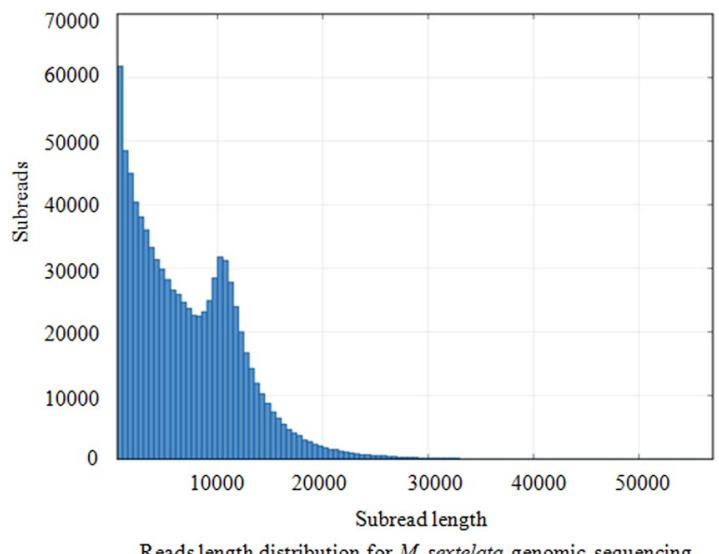

Figure 2. Horizontal coordinates represent sequencing read lengths. The bars correspond to the left vertical coordinate, which shows the read number relevant to each read length.

was conducted, and its genome was assembled. Additionally, the protein-coding genes, gene components, related biological functions, and secondary metabolite gene clusters were analyzed. Finally, the gene expression in several species of Morchella was compared in this study.

\section{Results}

DNA extraction and quality test. Approximately $16.57 \mu \mathrm{g}$ of DNA was extracted from the fresh Morchella strain. The quality test results showed that the purity (A260/A280) of DNA from the sample was 1.87, and the band of DNA on the gel after electrophoresis was clear, indicating that there was no protein, genomic, and other contamination. Thus, the DNA sample from the Morchella sample was sufficient for further analyses (Fig. S2). Before starting further analyses, the internal transcribed spacer (ITS) of DNA was sequenced, and ITS identification was performed by comparing the sequence to the sequences of known fungi in NCBI GenBank. Consequently, the Morchella strain was identified and confirmed as M. sextelata.

Second-generation genome sequencing and assembly. Illumina PE150 sequencing generated $2,985 \mathrm{Mb}$ raw reads, and 2,600 $\mathrm{Mb}$ clean reads were retained after the quality-control steps (data not shown). Then, the $M$. sextelata genome size was estimated to be $58.77 \mathrm{Mb}$ after revision by K-mer spectrum analysis with $\mathrm{K}-\mathrm{mer}=15$ and depth 27.36 . The heterozygous rate was $0.10 \%$, and the repeat rate was $36.09 \%$ (Table S1).

The clean reads were de novo assembled, and their scaffolds and contigs were analyzed. Notably, the generated results were only used to provide a reference for the following PacBio RSII third-generation analysis. Therefore, not all data are shown.

Third-generation genome sequencing and assembly. The raw reads from PacBio RSII were filtered by removing the reads with low quantity, and 869,182 clean reads were ultimately obtained (Table S2). The mean concordance of reads was 0.84 (Fig. 1), the mean read length was 6,626 bp and the N50 read length was 10,165 bp (Fig. 2). 


\begin{tabular}{|l|l|l|l|l|l|}
\hline Sample ID & Contig & Max Length (bp) & N50 Length (bp) & Total Length (bp) & Sequence GC (\%) \\
\hline M. sextelata & 59 & $4,823,818$ & $1,569,782$ & $52,925,331$ & 47.37 \\
\hline
\end{tabular}

Table 1. The contig statistics of the assembled genome of $M$. sextelata. Note: N50 length indicates the length of the contig that localized to $50 \%$ of the total contig length when the contigs are arranged from longer to shorter sizes.

\begin{tabular}{|l|l|}
\hline M. sextelata & Number or content \\
\hline Genome size (bp) & $52,925,331$ \\
\hline Gene number & 9,550 \\
\hline Gene total length (bp) & $13,107,305$ \\
\hline GC content in genes (\%) & 53.53 \\
\hline Gene content in genome (\%) & 24.77 \\
\hline Gene average length (bp) & 1,372 \\
\hline Internal gene length (bp) & $39,818,026$ \\
\hline Internal gene GC content (\%) & 45.34 \\
\hline Internal gene content in genome (\%) & 75.23 \\
\hline
\end{tabular}

Table 2. The summary of the final genome size and protein-coding gene annotation.

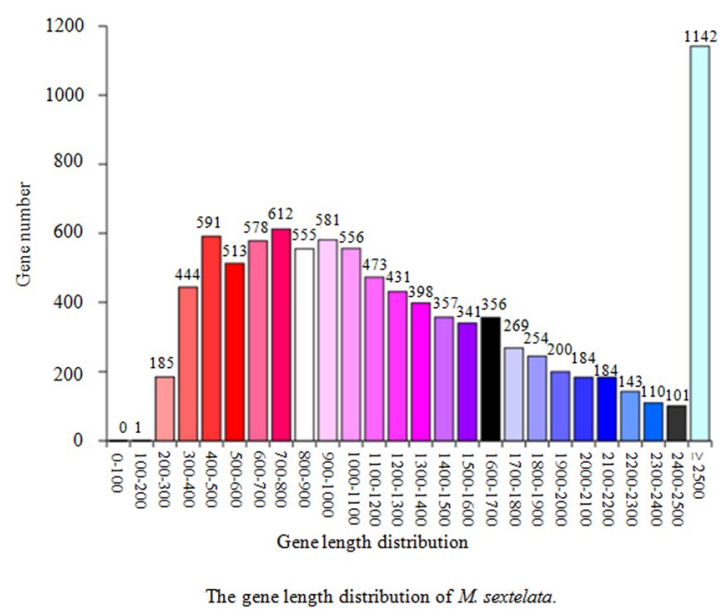

Figure 3. The horizontal coordinate represents the length of protein-coding genes, and the vertical coordinate shows the number of protein-coding genes. The number written on the top of the bar is the number of genes within each range of gene lengths.

The final clean reads from the third-generation PacBio RSII sequencing data were assembled, and a total of 59 contigs with N50 lengths of 1,569,782 bp were identified. The GC content of the whole-genome sequence was $47.37 \%$. Finally, the whole genome was generated, and the estimated size was 52,925,331 bp (approximately $52.93 \mathrm{Mb}$ ) (Table 1). This was smaller than the initially predicted size of $58.77 \mathrm{Mb}$ by the Illumina 150 system.

This Whole Genome Shotgun project has been deposited at DDBJ/ENA/GenBank under the accession SDUU0000000. The version described in this paper is version SDUU01000000.

Genome annotations. The protein-coding genes in the genome of M. sextelata were de novo predicted using the Augustus 2.7 program. A total of 9,550 protein-coding genes with a total length of $13,107,305 \mathrm{bp}$, accounting for $24.77 \%$ of the whole genome $(52,925,331 \mathrm{bp})$, were predicted. The lengths of the protein-coding genes mostly ranged from 300 to $1,700 \mathrm{bp}$ and were $1,372 \mathrm{bp}$ on average. The internal gene length was $39,818,026 \mathrm{bp}$, which accounted for $75.23 \%$ of the whole genome (Fig. 3) (Table 2).

Genome component predictions. The interspersed repetitive sequences (IRSs) and the tandem repeats (TRs) were determined. IRSs contain short interspersed nuclear elements (SINEs) and long interspersed nuclear elements (LINEs), with the latter often showing transposition activity. In M. sextelata, a total of 15,485 TRs with repeat sizes of 1-1,894 bp, 10,026 minisatellite DNAs with repeat sizes of 10-60 bp and 2,702 microsatellite DNAs with repeat sizes of $2-6$ bp were predicted (Table S3).

Noncoding RNAs (ncRNAs) play essential roles in biological processes, although they do not transcribe proteins. The results showed that there were $445 \mathrm{tRNAs}$ with an average length of $85 \mathrm{bp}$ in the $M$. sextelata genome. 


\begin{tabular}{|l|l|}
\hline M. sextelata & Enriched gene number \\
\hline NR & 6009 \\
\hline Swiss-Prot & 2702 \\
\hline KEGG & 5839 \\
\hline KOG & 2148 \\
\hline TCDB & 324 \\
\hline GO & 5876 \\
\hline PHI & 810 \\
\hline DFVF & 635 \\
\hline P450 & 61 \\
\hline Secretary protein & 542 \\
\hline CAZy & 290 \\
\hline
\end{tabular}

Table 3. The summary of gene function annotation in different databases. *NR: non-redundant protein database, KOG: eukaryotic orthologous groups, TCDB: transporter classification database, PHI: pathogen host interactions, DFVF: database of fungal virulence Factors, CAZy: carbohydrate-active enzymes database.

Compared to the amount of tRNA, the amounts of rRNA and snRNA were much lower, only 55 and 30, respectively. However, no rRNAs, sRNAs or miRNAs were predicted (Table S4).

Gene function. The functions of genes were predicted by different databases. The number of genes enriched in each functional classification is shown in Table 3. Generally, many more coding genes were enriched in Kyoto encyclopedia of genes and genomes (KEGG) and gene ontology (GO) compared to other databases.

In the GO analysis, a total of 5,876 genes were enriched. Among them, 2,184 genes were associated with cell, the same number of genes contributed to cell part, and 1,024 genes were enriched in organelle. A total of 3,381 genes were predicted to have binding abilities. The catalytic activity and transporter activity for 2,986 and 389 genes were predicted. Various biological processes were predicted to be mediated by the genes of M. sextelata. For instance, 3,335, 3,239, and 859 genes possibly participate in cellular process, metabolic process, and localization, respectively (Fig. S3).

KEGG pathway analysis was conducted, and the enrichment of genes in different processing, metabolism, and organismal systems was annotated as shown in Fig. S4. The results showed that 203 genes were enriched in the pathway of infectious disease, 110 and 91 genes were enriched in the signaling of cancers and neurodegenerative disease, and 56 genes were enriched in the process correlated with endocrine and metabolic disease.

The genes were also classified based on COG functional database. The majority of annotated genes (331) were predicted to have a general function only. The top three enriched categories were 1. posttranslational modification, protein turnover, chaperones (240 genes); 2. translation, ribosomal structure and biogenesis (219 genes); and 3. amino acid transport and metabolism (161 genes) (Fig. S5).

NR is a database established by NCBI for the annotation of a wide range of information, including species details. Here, most of the genes were annotated to be nonredundant proteins in Tuber melanosporum, 146 of the genes were annotated in Podospora anserine, and 84 genes were annotated in Pseudogymnoascus pannorum. The top 20 predicted nonredundant protein species are shown in Fig. S6.

Based on the above gene annotation and function analysis, the genomic map was drawn and is shown in Fig. 4.

In addition, the function of proteins was classified using TCDB. As shown in Fig. S7, most transporter proteins were enriched in primary active transporters, up to 120 . Next, 111 proteins were annotated as electrochemical potential-driven transporters, of which 55 were enriched in channels and pores.

There were 51 genes annotated in the endoplasmic reticulum retrieval protein 1 (Rer1; putative heavy metal transporter) family, 30 genes in the major facilitator superfamily (MFS), and 28 genes in the $\mathrm{H}^{+}-$or $\mathrm{Na}^{+}$-translocating NADH dehydrogenase (NDH) family. Additionally, some genes were predicted to be members of transporter families, such as the genes A3238 and A0956 in the ammonia transporter channel (Amt) family, A2965 and A3464 in the CorA metal ion transporter (MIT) family, and A8387, A8696, A9300, A1158, A1940, A4798 in the drug/metabolite transporter (DMT) Superfamily.

The CAZy database includes glycoside hydrolases (GHs), glycosyltransferases (GTs), polysaccharide lyases (PLs), carbohydrate esterases (CEs), and auxiliary activities (AAs). When the genes of M. sextelata were annotated by the CAZy database, GHs were the most abundant enzymes, as 159 genes were predicted to be GHs. As shown in Table 4, 57 of the genes were predicted to code carbohydrate modules (CBMs), while 44 of the genes were estimated to be AAs. In addition, the functions of $M$. sextelata genomes were also annotated by the Pfam and Swiss-Prot databases (data not shown).

The secondary metabolite gene clusters were analyzed. A total of 10 clusters, including 4 terpenes, $1 \mathrm{nps}, 1$ tlpks and 4 other clusters, were identified. The gene numbers for each cluster are marked on the blue bar (Fig. 5, Table 5 and Table 6). Additionally, the products of the gene clusters t1pks (polyketide synthase) and nrps (nonribosomal peptide synthase) were analyzed, and the chemical structures were predicted as shown in Fig. 6 .

In the effector analysis, the cytochrome P450 database was BLAST searched to annotate the genes encoding fungal cytochromes. The results showed that 62 of the genes were predicted to be P450s distributed in 6 classes. Specific gene IDs are provided in Table 7. 

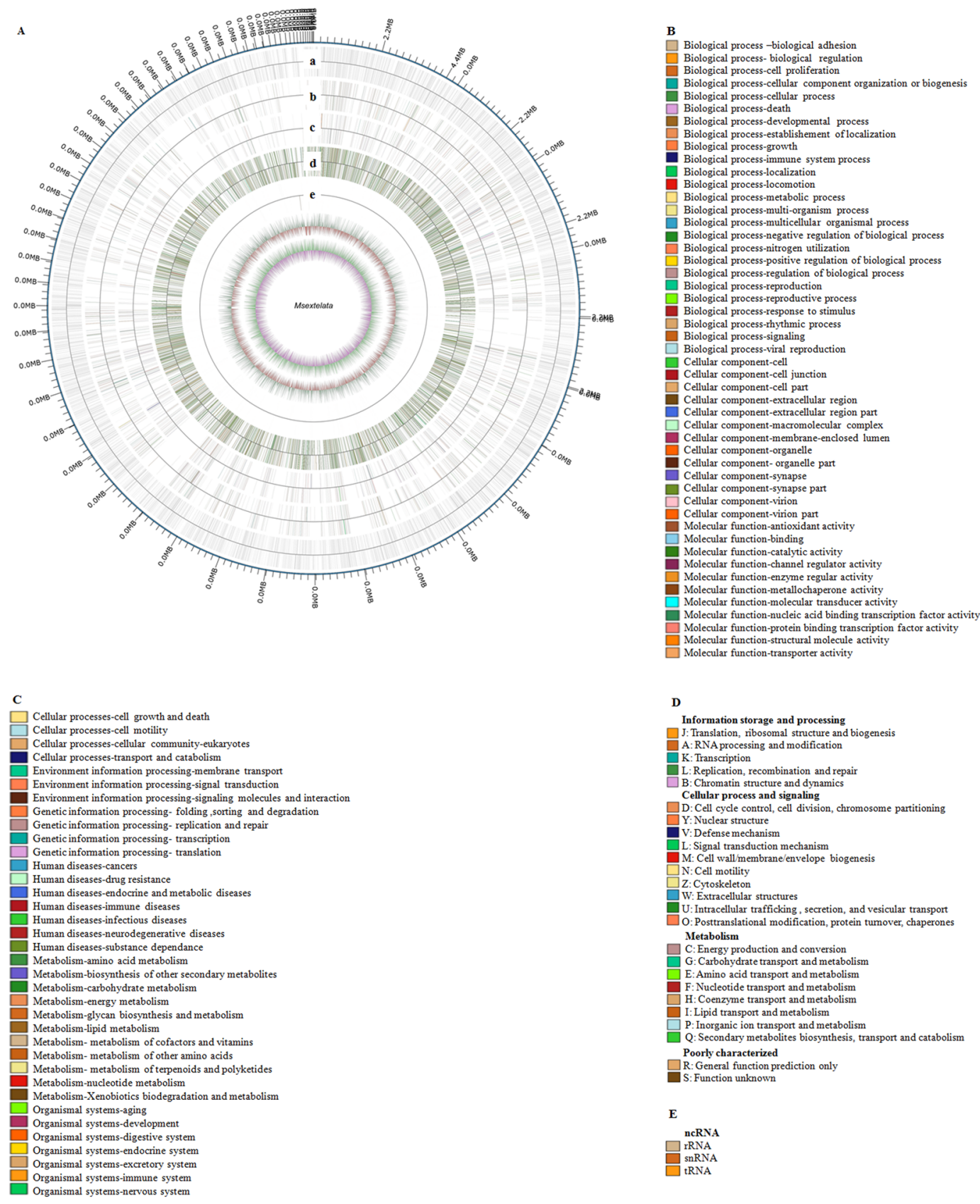

Figure 4. (A) The genetic map of M. sextelata. The outer ring is the gene position on the M. sextelata sequence. The ring (a) represents the information of coding gene positions. (b-d) represent the information of gene functions in the KOG, KEGG, and GO databases, respectively. (e) shows the ncRNA information. The outside ring is the positive chain, and the inner ring is the negative chain. The colors correspond to different functional characteristics predicted in the GO, KEGG, and KOG databases and different ncRNAs. (B) GO legend for (A). (C): KEGG legend for (A). (D) KOG legend for (A). (E) ncRNA legend for (A).

\begin{tabular}{|l|l|}
\hline CAZ__class & Match number \\
\hline CBM & 57 \\
\hline CE & 13 \\
\hline GH & 159 \\
\hline GT & 41 \\
\hline PL & 20 \\
\hline AA & 44 \\
\hline
\end{tabular}

Table 4. Summary of Carbohydrate-Active Enzymes database annotation. 
Eclusters_number $\mathbf{\text { gene_number }}$

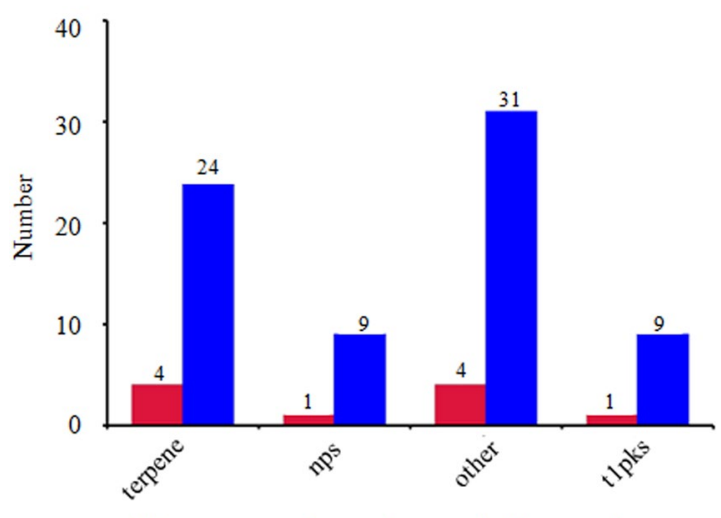

The summary of secondary metabolite gene clusters.

Figure 5. The horizontal coordinate represents the gene cluster name, and the vertical coordinate shows the number of gene clusters and genes in each cluster. Blue bars correspond to the gene number in each gene cluster, while red bars represent the number of clusters.

\begin{tabular}{|l|l|l|}
\hline Clusters & Cluster number & Gene number \\
\hline terpene & 4 & 24 \\
\hline nrps & 1 & 9 \\
\hline tlpks & 1 & 9 \\
\hline other & 4 & 31 \\
\hline
\end{tabular}

Table 5. The summary of secondary metabolite gene clusters.

\begin{tabular}{|l|l|l|l|}
\hline & Clusters & Contigs & Genes \\
\hline 1 & terpene & Contig3 & A6295; A6296; A6297; A6298 \\
\hline 2 & other & Contig4 & A7647; A7648; A7650; A7651; A7652; A7653; A7654; A7655; A7656 \\
\hline 3 & other & Contig8 & A9156; A9157; A9158; A9159; A9160; A9161; A9162; A9163 \\
\hline 4 & terpene & Contig11 & A0451; A0452; A0453; A0454; A0455 \\
\hline 5 & terpene & Contig14 & A1287; A1288; A1289; A1290; A1291; A1292; A1293; A1295; A1296; A1297 \\
\hline 6 & tlpks & Contig20 & A3426; A3427; A3428; A3429; A3430; A3431; A3432; A3433; A3434 \\
\hline 7 & nrps & Contig27 & A4555; A4556; A4557; A4558; A4559; A4560; A4561; A4562; A4563 \\
\hline 8 & other & Contig28 & A4609; A4610; A4611; A4612; A4613; A4614; A4615; A4616 \\
\hline 9 & other & Contig33 & A5824; A5825; A5826; A5827; A5828; A5829 \\
\hline 10 & terpene & Contig36 & A6021; A6022; A6023; A6024; A6025 \\
\hline
\end{tabular}

Table 6. The statistics of secondary metabolite gene clusters.

Comparative genomic analysis. First, the genomic assembly results for M. sextelata, M. septimelata ${ }^{9}, M$. eximia ${ }^{9}, M$. importuna ${ }^{3}$, and $M$. conica $^{10}$ were compared and are summarized in Table 8.

Then, the shared and specific genes in different species of Morchella were analyzed as shown in Fig. 7. The results showed that $M$. eximia exhibited the highest specific gene families with 7,347, and $M$. sextelata also showed 1,139 specific gene families, which is much higher than the number of specific gene families in other species, ranging from 371 to 837 . On the other hand, the number of shared gene families was 3,717 .

Furthermore, the dispensable genes, which are all genes except the core genes, in each strain were analyzed, and a heatmap is shown in Fig. 8. Consistent with the results in the Venn diagram, M. eximia (QMFK00000000.1) showed a significant difference compared to other Morchella. Additionally, a large number of specific genes were found in M. sextelata. For example, the gene A8276 specifically expressed in M. sextelata was predicted to be involved in bladder cancer, thyroid cancer, and acute myeloid leukemia. Interestingly, the gene A5703 was also functionally related to bladder cancer, thyroid cancer, and acute myeloid leukemia in these Morchella species.

In-depth analysis revealed that the shared genes annotated to encode PLs were A5192, A3285, A5387, A5018, A5042, and A0650 in these Morchella species. Comparatively, M. sextelata was found to have 14 specific genes encoding PLs, for example, A2625, A4964, and A7924 (Table S5), suggesting some unique biological features of 
A<smiles>[R]CC(O)C([R])C(O)NC([R])C=O</smiles>

B<smiles>[R]C(C=O)NC(=O)C([R])NC(=O)[C@H](C)NC(=O)C([R])N</smiles>

The estimated structure of the products of gene clusters.

Figure 6. A: The predicted structure of the product of gene cluster 6; B: the predicted structure of the product of gene cluster 7 .

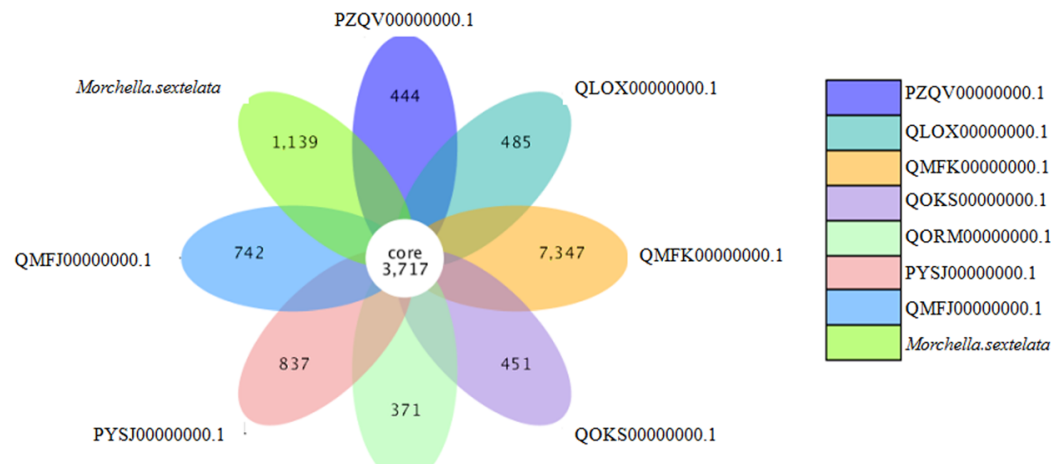

QORM00000000.1

Venn diagram showing specific and shared gene families.

Figure 7. PZQV00000000.1 represents Morchella conica strain CCBAS932, QLOX00000000.1 and PYSJ00000000.1 represent Morchella septimelata strain MG91, QMFK00000000.1 represents Morchella eximia MG90, QOKS00000000.1 represents Morchella importuna M04M26, QORM00000000.1 represents Morchella importuna M04M24, and QMFJ00000000.1 represents Morchella septimelata MG113. Morchella sextelata is the strain studied in this work. A total of 3,717 genes written in the center indicate the gene number shared in these different Morchella species (or strains), while the numbers described in the diagram show the genes specifically expressed in each Morchella species (or strain).

\begin{tabular}{|l|l|l|l|}
\hline P450 class & Class name & Gene number & Gene \\
\hline 1 & P450, CYP52 & 7 & A7570, A0041, A7809, A2469, A1467, A3417, A4602 \\
\hline 2 & E-class P450, CYP2D & 2 & A7321, A1285 \\
\hline 3 & Undetermined & 8 & A8534, A8641, A1943, A1303, A0047, A0110, A1004, A1375 \\
\hline 4 & Cytochrome P450 & 11 & $\begin{array}{l}\text { A6397, A8907, A2892, A7709, A0184, A3635, A4884, A6462, A2432, } \\
\text { A5836, A5236 }\end{array}$ \\
\hline 5 & E-class P450, group I & 25 & $\begin{array}{l}\text { A2224, A0822, A0168, A1345, A7129, A6827, A6624, A9296, A7194, } \\
\text { A9622, A2360, A3045, A8405, A6914, A1184, A1955, A9488, A5674, }\end{array}$ \\
\hline 6 & E-class P450, group IV & 8 & A1869, A6522, A6749, A0104, A0103, A3061, A4737, A5331 \\
\hline
\end{tabular}

Table 7. The statistics of $\mathrm{P} 450$ genes.

M. sextelata. PLs play important roles in the metabolism of polysaccharides, which have been reported to have antitumor activity ${ }^{4}$. This indicates that comparative analysis can provide important data for conducting extensive studies on the biological function of M. sextelata and other Morchella species. (Supplementary data, dispensable matrix). 


\begin{tabular}{|c|c|c|c|c|c|c|c|c|c|c|c|}
\hline & Species & Strain & $\begin{array}{l}\text { Assembly } \\
(\mathrm{Mb})\end{array}$ & GC\% & Scaffolds & Contigs & N50 & $\begin{array}{l}\text { Genome } \\
\text { coverage }\end{array}$ & INSDC & Sequencing & Assembly method \\
\hline 1 & Morchella septimelata & MG91 & 49.62 & 47.1 & 3707 & 5121 & 28400 & $58 \mathrm{x}$ & QLOX00000000.1 & Illumina & platanus version v. 1.2.1 \\
\hline 2 & Morchella eximia & MG90 & 73.46 & 46.0 & 7793 & 10613 & 26474 & $57 \mathrm{x}$ & QMFK00000000.1 & Illumina & platanus version v. 1.2.1 \\
\hline 3 & Morchella importuna & M04 M26 & 51.08 & 47.3 & 106 & 110 & 958716 & $298 x$ & QOKS00000000.1 & Illumina HiSeq & AllPaths v. 44849 \\
\hline 4 & Morchella conica & CCBAS932 & 48.21 & 47.2 & 540 & 2145 & 52248 & $67.8 \mathrm{x}$ & PZQV00000000.1 & Illumina & AllPathsLG v. R47710 \\
\hline 5 & Morchella importuna & M04 M24 & 48.86 & 47.0 & 323 & 504 & 362388 & $210 x$ & QORM00000000.1 & Illumina HiSeq & AllPaths v. 44849 \\
\hline 6 & Morchella septimelata & MG91 & 49.96 & - & 5231 & 5241 & 37765 & $151.17 \mathrm{x}$ & PYSJ00000000.1 & Illumina GAIIx & SPAdes v. 3.0 \\
\hline 7 & Morchella septimelata & MG113 & 51.40 & - & 9172 & 11637 & 28426 & $88 \mathrm{x}$ & QMFJ00000000.1 & Illumina & platanus version v. 1.2.1 \\
\hline
\end{tabular}

Table 8. Genomic assembly statistics of different species of Morchella.

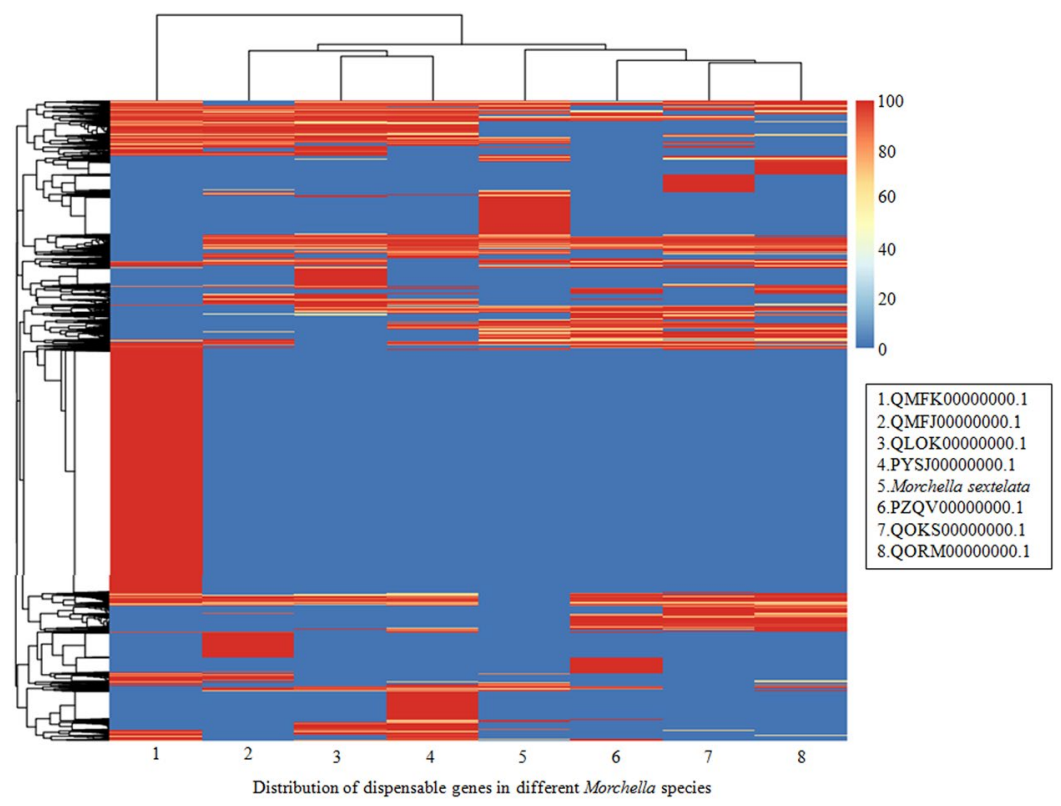

Figure 8. Left: the clustering tree of dispensable genes. Top: the clustering tree of samples. Middle: the expression level of dispensable genes in Morchella species (or strains) with different identity values, which are described with different colors, as shown on the upper right.

\section{Discussion}

The genome sequencing in this study provides the whole-genome sequence of $M$. sextelata and its related gene annotation for the first time. This study may provide important data for evaluating Morchella species, improving culture techniques by mediating mating behavior, and discovering biologically active compounds. It is important not only for meeting the increasing demand for M. sextelata but also for further research on M. sextelata.

The comparative genomic analysis of M. sextelata and other Morchella species, M. conica, M. importuna, M. septimelata, and M. eximia, was carried out in this study. To provide additional useful information, the gene annotation file generated in this study was also uploaded and may provide useful data for further research on the differences between various Morchella species and their biological functions in the future.

The biological roles of Morchella have been reported in recent years. The potential neuritogenic activity of the aqueous extract from $M$. importun ${ }^{11}$, immunomodulatory activity of the exopolysaccharides extracted from $M$. conica $^{12}$, and antitumor activity of polysaccharides isolated from $M$. esculenta were determined ${ }^{4}$. In addition, a polysaccharide from $M$. conica was found to inhibit $\mathrm{H}_{2} \mathrm{O}_{2}$-induced oxidative stress in human embryonic kidney $293 \mathrm{~T} \mathrm{cells}^{13}$. In addition to these, many other biological functions of the compounds obtained from different Morchella have been identified, suggesting the great value of further studies of Morchella.

\section{Materials and Methods}

M. sextelata sample. The self-mated $M$. sextelata spores were cultured in sandy soil in a plastic greenhouse with controlled light, temperature and humidity in Shagedu town located at the eastern end of the Ordos Plateau in Inner Mongolia, China. The M. sextelata isolate we cultured usually had darker caps and larger body sizes. The average size was approximately $15 \mathrm{~cm}$ high. It is typically planted from October to November and is picked up from April to May in the next year. Since Morchella grows at low temperatures, the weather of Inner Mongolia is very suitable. 


\section{DNA extraction and quality test.}

(1) A fresh Morchella sample was collected and ground completely in a mortar and pestle precooled with liquid nitrogen.

(2) The sample was then transferred into a 50-ml tube containing $20 \mathrm{ml} \mathrm{GP1}$ and $500 \mu \mathrm{l}$ mercaptoethanol and mixed thoroughly by shaking in a $55^{\circ} \mathrm{C}$ water bath. The sample was centrifuged at $12,500 \mathrm{rpm}$ for $8 \mathrm{~min}$ (GP1 buffer: RK116-02).

(3) The supernatant was collected, and saturated phenol/chloroform/isoamyl alcohol (25:24:1) was added. Then, it was centrifuged after mixing at $12,500 \mathrm{rpm}$ for $8 \mathrm{~min}$.

(4) The supernatant was collected and mixed with chloroform/isoamyl alcohol. Then, the mixture was centrifuged at $12,500 \mathrm{rpm}$ for $8 \mathrm{~min}$ after vortexing.

(5) The supernatant was collected again in a 50- $\mathrm{ml}$ new tube, and isopropanol was added and mixed well. The sample was placed at $-20^{\circ} \mathrm{C}$ to allow precipitation for a few minutes. Then, the mixture was centrifuged $(12,500 \mathrm{rpm}, 8 \mathrm{~min})$ and the supernatant was removed.

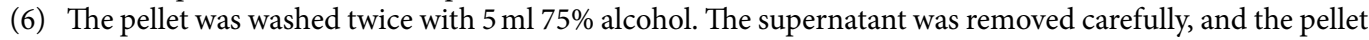
was dried.

(7) A total of $100 \mu \mathrm{lddH} \mathrm{d}_{2} \mathrm{O}$ was added to dissolve the DNA sample. Then, $2 \mu \mathrm{l}$ RNase was added to digest RNA, and the sample was then shaken several times and incubated at $37^{\circ} \mathrm{C}$ for $15 \mathrm{~min}$.

(8) Finally, the DNA sample was purified with the PowerClean Pro DNA.

(9) Clean-Up Kit (No. 12997-50), and $154 \mu \mathrm{l}$ of DNA sample with a concentration of $107.60 \mathrm{ng} / \mu \mathrm{l}$ was obtained. A total of $16.570 \mu \mathrm{g}$ DNA was prepared for further analysis.

To ensure that the harvested DNA sample was suitable for further analysis and that there was no contamination, the quality of the harvested DNA was examined by agarose gel electrophoresis.

Genome sequencing and assembly via a second-generation system. First, the DNA was randomly cut into $350 \mathrm{bp}$ fragments by Covaris ultrasonic processor. The fragments were modified with end-repairing and the addition of A tail and sequencing adaptors and were purified and PCR amplified to generate a $350 \mathrm{bp}$ long fragment library. Next, the library was initially quantified by Qubit 2.0, and the insert size of fragments was tested by Agilent 2100. Then, the effective concentration of the library was examined by q-PCR to ensure that the quality of the $350 \mathrm{bp}$ library was fit for further sequencing analysis. Subsequently, the genome of the Morchella sample in the $350 \mathrm{bp}$ libraries was sequenced by the second-generation Illumina PE150 system. The generated reads were filtered by several steps to obtain the clean reads, which were then analyzed through K-mer $(\mathrm{K}-\mathrm{mer}=15)$ to estimate the genome size.

The genome for the M. sextelata sample was obtained by assembling the clean data from the Illumina PE150 system by SOAP de novo (version 2.04) ${ }^{14}$, SPAde ${ }^{15}$ and $\mathrm{ABySS}^{16}$ software and subsequently integrating the genome sequence with CISA ${ }^{17}$ software. The initially assembled genome was optimized and supplemented by the gapclose (version 1.12) program. Consequently, the result of the assembly was statistically analyzed by scaffolds and contigs after excluding fragments $<500 \mathrm{bp}$. Finally, the GC content and coverage depth were analyzed to determine whether there was sequence contamination and repeat sequences.

All of the results obtained from the Illumina PE150 system were used as the survey for the PacBio RSII third-generation sequencing and further analyses as a source of genomic information.

Genome sequencing and assembly via a third-generation system. After the survey was performed using the Illumina PE150 system, another part of the DNA sample was cut into $20 \mathrm{~Kb}$ fragments by Covaris g-TUBE, and the DNA damage and ends of the DNA were repaired. Second, both ends of the fragments were joined with a barcode. Next, these fragments were purified with AM pure PB to generate $20 \mathrm{~Kb}$ SMRT Bell libraries. The fragments $20 \mathrm{~Kb}$ in length were then screened through BluePippin and purified with AMPure PB beads. Subsequently, the library was quantified by Qubit 2.0, and the insert size of fragments was examined by Agilent 2100. After that, the genome of $M$. sextelata in the $20 \mathrm{~Kb}$ SMRT Bell libraries was sequenced by the PacBio RSII third-generation sequencing system. The obtained raw reads were filtered to obtain the clean reads.

The clean reads were assembled using SMRT Link v5.1.0 software (https://www.pacb.com/support/ software-downloads/ $/)^{18,19}$ by consulting the results from the second-generation sequencing data. De novo assembly was carried out based on the results from the Illumina PE150 system since no reference genome of other Morchella was available. Then, the genome obtained from the PacBio RSII third-generation sequencing system was used for all of the further analyses, including the prediction of genome size, genome annotation, genome component and gene function.

Such an assembly that combines second- and third-generation sequencing data can revise and decrease the interference of abnormal GC contents, high repetition and hybridity. Therefore, it can improve the integrity and uniformity of the generated genome sequence. It is particularly applicable for complex fungi such as Morchella, which lack comparable genomic information, such as reference sequences, GC contents, and repetitive sequences.

Both sequencing procedures were performed using the Illumina HiSeq. 4000 sequencing platform at Beijing Novogene Bioinformatics Technology Co., Ltd.

Genome annotations. For M. sextelata, by default, we performed de novo prediction using the Augustus 2.7 program $^{20}$ to obtain the protein-coding genes.

Genome component predictions. Genome component prediction included the prediction of the coding genes, the repetitive sequences and the ncRNA. The interspersed repetitive sequences were predicted using 
RepeatMasker (http://www.repeatmasker.org/) ${ }^{21}$. The tandem repeats were analyzed by tandem repeats finder ${ }^{22}$. NcRNAs include transfer RNAs (tRNAs), ribosome RNAs (rRNAs), small RNAs (sRNAs), small nuclear RNAs (snRNAs), and microRNAs (miRNAs). The tRNA genes were predicted by tRNAscan-SE ${ }^{23}$ (Version 1.3.1), while the rRNA genes were analyzed by rRNAmmer ${ }^{24}$ (Version 1.2). The genes for sRNA, snRNA and miRNA were BLAST searched against the Rfam 3 database, annotated ${ }^{25}$, and then confirmed by cmsearch (Version 1.1rc4).

Gene function. We mainly used the $\mathrm{GO}^{26}$, KEGG ${ }^{27,28}$, KOG (http://www.ncbi.nlm.nih.gov/COG/), $\mathrm{NR}^{29}$, $\mathrm{TCDB}^{30}, \mathrm{P} 450$, Pfam (http://pfam.xfam.org/), and Swiss-Prot ${ }^{31}$ databases to predict gene functions. A whole-genome BLAST search (E-value less than 1e-5, minimal alignment length percentage larger than 40\%) was performed against the above databases. The secretory proteins were predicted by the Signal $\mathrm{P}$ database ${ }^{32}$ (version 4.1) and TMHMM (version 2.0c). Moreover, we analyzed the secondary metabolite gene clusters with antiSMASH ${ }^{33}$ (version 2.0.2). Additionally, carbohydrate-active enzymes were predicted by the CAZy ${ }^{34}$ database.

Comparative genomics analysis. The core genes and specific genes of the reference genomes, $M$. septimelata, M. eximia, M. importuna, and $M$. conica, were compared to the genome of $M$. sextelata. The core genes and specific genes were analyzed by CD-HIT rapid clustering of similar proteins software with a threshold of $50 \%$ pairwise identity and 0.7 length difference cutoff in amino $\operatorname{acid}^{35-37}$. Then, the Venn diagram and heatmap were drawn to show their relationships and specificities among the samples.

\section{Data availability}

The assembled genome sequence of $M$. sextelata has been provided to NCBI with the SRA project ID PRJNA560199, and all raw data are available.

Received: 15 November 2018; Accepted: 20 September 2019;

Published online: 25 October 2019

\section{References}

1. He, S., Zhao, K., Ma, L., Yang, J. \& Chang, Y. Effects of different cultivation material formulas on the growth and quality of Morchella spp. Saudi J. Bio. Sci. 25, 719-723 (2018).

2. Irfan, M., Yang, S., Yuxin, L. \& Sun, J. X. Genetic diversity analysis of Morchella spp. by RAPD. Mol. Bio. Res. Commun. 6, 27-31 (2017).

3. Liu, W., Chen, L. F., Cai, Y. L., Zhang, Q. Q. \& Bian, Y. B. Opposite polarity monospore genome de novo sequencing and comparative analysis reveal the possible heterothallic life cycle of Morchella importuna. Int. J. Mol. Sci. 25, 1-22 (2018).

4. Liu, C. et al. Characteristics and antitumor activity of Morchella esculenta polysaccharide extracted by pulsed electric field. Int. J. Mol. Sci. 17(6), E986 (2016).

5. He, P., Wang, K., Cai, Y. \& Liu, W. Live cell confocal laser imaging studies on the nuclear behavior during meiosis and ascosporogenesis in Morchella importuna under artificial cultivation. Micron. 101, 108-113 (2017).

6. Du, X. H., Zhao, Q. \& Yang, Z. L. A review on research advances. issues, and perspectives of morels. Mycology. 6, 78-85 (2015).

7. Richard, F. et al. True morels (Morchella, Pezizales) of Europe and North America: evolutionary relationships inferred from multilocus data and a unified taxonomy. Mycologia. 107, 359-382 (2015).

8. Du, X. H. et al. Mixed-reproductive strategies, competitive mating-type distribution and life cycle of fourteen black morel species. Sci. Rep. 7, 1493 (2017).

9. Li, H. Y. et al. The genome sequences of 90 mushrooms. Sci. Rep. 89, 9982 (2018).

10. Murat, C. et al. Pezizomycetes genomes reveal the molecular basis of ectomycorrhizal truffle lifestyle. Nat Ecol Evol. 2(12), 1956-1965 (2018).

11. Xiong, C. et al. The potential neuritogenic activity of aqueous extracts from Morchella importuna in rat pheochromocytoma cells. Food Sci. Biotechnol. 26, 1685-1692 (2017).

12. Su, C. A. et al. Isolation and characterization of exopolysaccharide with immunomodulatory activity from fermentation broth of Morchella conica. Daru. 21, 5 (2013).

13. Xu, N., Lu, Y., Hou, J. M., Liu, C. \& Sun, Y. H. A polysaccharide purified from Morchella conica Pers. prevents oxidative stress induced by $\mathrm{H} 2 \mathrm{O} 2$ in human embryonic kidney (HEK) 293T cells. Int. J. Mol. Sci. 19, E4027 (2018).

14. Li, R., Li, Y., Kristiansen, K. \& Wang, J. SOAP: short oligonucleotide alignment program. Bioinformatics. 24, 713-714 (2008).

15. Bankevich, A. et al. SPAdes: a new genome assembly algorithm and its applications to single-cell sequencing. J. Comput. Biol. 19, 455-477 (2012).

16. Simpson, J. T. et al. ABySS: a parallel assembler for short read sequence data. Genome Res. 19, 1117 (2009).

17. Lin, S. H. \& Liao, Y. C. CISA: contig integrator for sequence assembly of bacterial genomes. PloS one. 8, e60843 (2013).

18. Ardui, S., Ameur, A., Vermeesch, J. R. \& Hestand, M. S. Single molecule real-time (SMRT) sequencing comes of age: applications and utilities for medical diagnostics. Nucleic Acids Res. 46, 2159-2168 (2018).

19. Reiner, J. et al. Cytogenomic identification and long-read single molecule real-time (SMRT) sequencing of aBardet-Biedl Syndrome 9(BBS9) deletion. NPJ. Genom. Med. 3, 1-5 (2018).

20. Stanke, M., Diekhans, M., Baertsch, R. \& Haussler, D. Using native and syntenically mapped cDNA alignments to improve de novo gene finding. Bioinformatics. 24, 637-644 (2008).

21. Saha, S., Bridges, S., Magbanua, Z. V. \& Peterson, D. G. Empirical comparison of ab initio repeat finding programs. Nucleic Acids Res. 36, 2284-2294 (2008).

22. Benson, G. Tandem repeats finder: a program to analyze DNA sequences. Nucleic Acids Res. 27, 573-580 (1999).

23. Lowe, T. M. \& Eddy, S. R. tRNAscan-SE: a program for improved detection of transfer RNA genes in genomic sequence. Nucleic Acids Res. 25, 0955-964 (1997).

24. Lagesen, K. et al. RNAmmer: consistent and rapid annotation of ribosomal RNA genes. Nucleic Acids Res. 35, 3100-3108 (2007).

25. Gardner, P. P. et al. Rfam: updates to the RNA families database. Nucleic Acids Res. 37, D136-140 (2009).

26. Ashburner, M. et al. Gene Ontology: tool for the unification of biology. Nat. Genet. 25, 25-29 (2000).

27. Kanehisa, M., Goto, S., Kawashima, S., Okuno, Y. \& Hattori, M. The KEGG resource for deciphering the genome. Nucleic Acids Res. 32, D277-D280 (2004).

28. Kanehisa, M. et al. From genomics to chemical genomics: new developments in KEGG. Nucleic Acids Res. 34, D354-D357 (2006).

29. Li, W., Jaroszewski, L. \& Godzik, A. Tolerating some redundancy significantly speeds up clustering of large protein databases. Bioinformatics. 18, 77-82 (2002). 
30. Milton, S. J., Vamsee, S. R., Dorjee, G. T. \& Västermark, A. The transporter classification database. Nucleic Acids Res. 42, D251-D258 (2014).

31. Amos, B. \& Rolf, A. The SWISS-PROT protein sequence database and its supplement TrEMBL in 2000. Nucleic Acids Res. 28, 45-48 (2000).

32. Petersen, T. N. et al. SignalP 4.0: discriminating signal peptides from transmembrane regions. Nat. Methods. 8, 785-786 (2011).

33. Medema, M. H. et al. antiSMASH: rapid identification, annotation and analysis of secondary metabolite biosynthesis gene clusters in bacterial and fungal genome sequences. Nucleic Acids Res. 39, W339-W346 (2011).

34. Cantarel, B. L. et al. The Carbohydrate-Active EnZymes database (CAZy): an expert resource for glycogenomics. Nucleic Acids Res. 37, D233-D238 (2009).

35. Jia, B. et al. CARD 2017: expansion and model-centric curation of the comprehensive antibiotic resistance database. Nucleic Acids Res. 45, D566-D573 (2017).

36. Kurtz, S. et al. Versatile and open software for comparing large genomes. Genome Biol. 5(2), R12 (2004).

37. Harris, R. S. Improved pairwise alignment of genomic DNA [M]. ProQuest (2007).

\section{Acknowledgements}

This work was supported by the "Young Innovative Entrepreneurs" foundation of "Prairie Talents" engineering of Inner Mongolia, China, No. NEI REN SHE FA (2017) 67 and the foundation from Inner Mongolia ZhongXing Agriculture and Animal Husbandry Development Co., Ltd. and Inner Mongolia Ao Dun Agricultural Technology Co., Ltd., China.

\section{Author contributions}

Qingshan-WANG and Baiyintala were mainly responsible for the culture and collection of samples for the experiments. Mei-HAN and Wuhanqimuge completed the related experiments, and Wuhanqimuge wrote the main manuscript. All authors reviewed the results and approved the final version of the manuscript.

\section{Competing interests}

The authors declare no competing interests.

\section{Additional information}

Supplementary information is available for this paper at https://doi.org/10.1038/s41598-019-51831-4.

Correspondence and requests for materials should be addressed to $\mathrm{W}$.

Reprints and permissions information is available at www.nature.com/reprints.

Publisher's note Springer Nature remains neutral with regard to jurisdictional claims in published maps and institutional affiliations.

(c) (i) Open Access This article is licensed under a Creative Commons Attribution 4.0 International License, which permits use, sharing, adaptation, distribution and reproduction in any medium or format, as long as you give appropriate credit to the original author(s) and the source, provide a link to the Creative Commons license, and indicate if changes were made. The images or other third party material in this article are included in the article's Creative Commons license, unless indicated otherwise in a credit line to the material. If material is not included in the article's Creative Commons license and your intended use is not permitted by statutory regulation or exceeds the permitted use, you will need to obtain permission directly from the copyright holder. To view a copy of this license, visit http://creativecommons.org/licenses/by/4.0/.

(c) The Author(s) 2019 